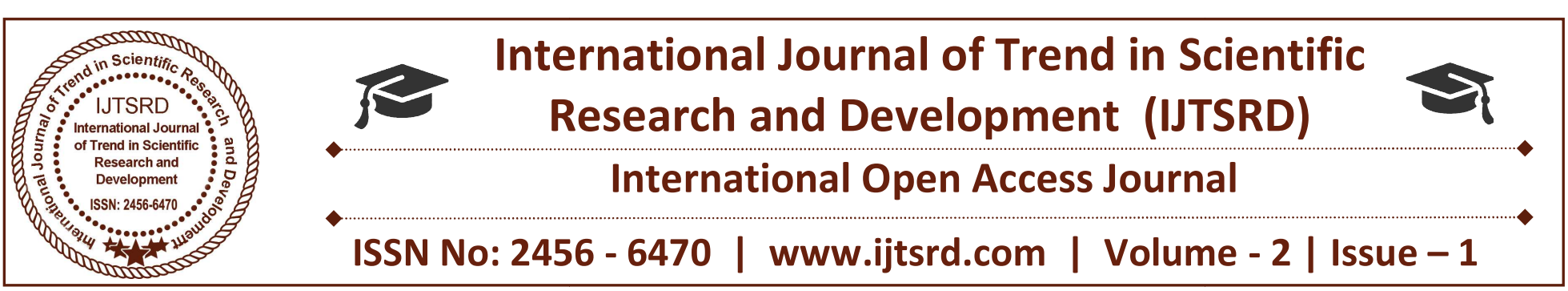

\title{
An Effective and Optimal Mobility Model and its Prediction in MANETs
}

\author{
Shashiraj Teotia \\ Research Scholar, IFTM University \\ Moradabad, Uttar Pradesh
}

\author{
Dr. Sohan Garg \\ Professor \& Director, SCRIET, CCS University \\ Meerut, Uttar Pradesh
}

\section{ABSTRACT}

In our research paper, we display an overview of different mobility models in ad hoc wireless networks. We demonstrate that gathering movement happens much of the time in specially appointed networks, and present another mobility model that is exceptionally effective and optimal for MANETs i.e. Optimal and Effective Mobility Model (OEMM) to speak to the relationship among portable hosts. OEMM can be promptly connected to numerous current applications. In addition, by appropriate selection of parameters, OEMM can be utilized to model a few mobility models which were beforehand proposed. One of the primary topics of this paper is to explore the effect of the mobility model on the execution of a particular network protocol or application. To this end, we have connected our OEMM model to two distinctive network protocol situations, bunching and directing, and have assessed network execution under various mobility designs and for various protocol usage. Not surprisingly, the outcomes demonstrate that distinctive mobility designs influence the different protocols in various ways. Specifically, the positioning of steering calculations is affected by the decision of mobility design.

Keyword: Mobility, multihop, distributed efficiency

\section{INTRODUCTION}

Ad hoc wireless networks will be networks which don't depend on a prior correspondence foundation. Or maybe, they keep up a dynamic interconnection topology between portable clients, regularly var multihoping. Specially appointed networks are relied upon to assume an undeniably essential part in future regular citizen and military settings where remote access to a wired spine is either ineffective or unthinkable. Specially appointed network applications extend from communitarian, appropriated versatile figuring to catastrophe recuperation (fire, surge, earthtremor), law implementation (swarm control, inquiry and protect) what's more, computerized war zone correspondences. Some key qualities of these frameworks are group coordinated effort of huge number of versatile units, restricted transmission capacity, the requirement for supporting sight and sound continuous activity and low dormancy access to appropriated assets (e.g., circulated database access for circumstance mindfulness in the war zone).

The hosts in a specially appointed network move as per different examples. Reasonable models for the movement designs are required in recreation with a specific end goal to assess framework and protocol execution. A large portion of the prior research on mobility designs depended on cell networks. Mobility pat-terns have been utilized to infer activity and mobility expectation models in the investigation of different issues in cell frameworks, for example, handoff, area administration, paging, enlistment, calling time, movement stack. As of late, mobility models have been investigated likewise in specially appointed networks. While in cell networks, mobility models are predominantly centered around singular developments since interchanges are point to point as opposed to among gatherings; in specially appointed networks, correspondences are frequently among groups which tend to arrange their developments (e.g., a fire fighters protect group in a fiasco recuperation circumstance). 
Consequently, the need emerges for creating proficient and sensible gathering mobility models.

Obviously, mobility models are application subordinate. Also, we expect that the different mobility examples will influence the execution of various network protocols in various ways. Subsequently, we are building up an adaptable mobility system which enables us to model diverse applications and network situations (e.g., individual and gathering; cell and impromptu, and so on.) and to recognize the effect of mobility on various situations. The proposed mobility system is called Optimal and Effective Mobility Model (OEMM) model. In the model, portable hosts are composed by bunches as indicated by their consistent connections. We ponder the effect of mobility on: (a) network topology availability and; (b) routing protocols. We utilize DSDV [18], AODV [17] and HSR [16] for the assessment and examination of routing plan execution. Next, as we trust that a clustering framework [9] can diminish the effect of topology changes on routing .we contemplate the mobility affect on cluster stability also.

This paper is composed as takes after. A study of mobility models in impromptu networks is given in area 2 . Area 3 concentrates on bunch mobility models. The new Optimal and Effective Mobility model is presented and a few mobility applications are depicted. The recreation comes about featuring the impact of gathering mobility models on availability, cluster stability and routing execution are given in segment 4 . Area 5 finishes up the paper.

\section{SURVEY OF EXISTING MOBILITY MODELS FOR MANETs:}

In a wireless network, mobile hosts (MHs) can move in a wide range of ways. Mobility models are usually used to dissect recently planned frameworks or protocols in both cell and impromptu remote networks. In cell remote networks, thinks about for mobility models not just go for depicting singular movement practices, for example, alters in course and speed, yet in addition consider the aggregate movement of the considerable number of mobiles with respect to a land region (cell) after some time. Models for specially appointed network mobility for the most part mirror the conduct of an individual versatile, or a gathering of mobiles. Be that as it may, there is no thought of aggregate development of all mobiles with reference to a specific "cell".
The most widely recognized model is the arbitrary walk model. It has been utilized by numerous creators, for example, Rubin [19], Zonoozi [21], Decker [8] and BarNoy [2]. The model portrays singular development in respect to cells. In this model, a versatile host moves from its present position to the following position haphazardly. The speed and bearing are picked consistently from the numerical reaches and individually. In a run of the mill Markovian model [2] for one dimensional arbitrary walk, a $\mathrm{MH}$ in cell is accepted to move to cells or to remain in cell with given transition probabilities. The irregular walk model has been utilized to research a wide arrangement of various framework parameters. For instance, Rubin utilizes the arbitrary development suspicion to get the mean cell stay time in the first place, at that point to determine numerous other framework measures. Zonoozi conducts an efficient following of the irregular development of a $\mathrm{MH}$. At every moment, he segments the entire region into a few districts as per past, present and next movement headings of a portable host. He scientifically gives the conditions for developments from the present area into the following locale. His following of mobility prompts the figuring of channel holding time and handover number. Decker portrays an individual $\mathrm{MH}$ with the mean span of remain in the present position and the likelihood of picking a moving way. A predesigned state-transit matrix can give the versatile host a movement congratulate tern, for example, proceeding onward an interstate, on boulevards or simply like an irregular passerby. In specially appointed remote portable networks, the mobility models concentrate on the individual movement conduct between mobility ages, which are the littlest eras in a reenactment in which a versatile host moves in a steady bearing at a consistent speed.

Numerous specialists utilize the arbitrary mobility model [14, 15, 21, and 20]. As indicated by this model, the speed and heading of movement in another time interim have no connection to their past qualities in the past age. This model can produce un-sensible portable conduct, for example, sharp turning or sudden ceasing.

A few creators utilize adjusted adaptations of the irregular mobility model. Basagni [3] portrays the development of $\mathrm{MHs}$ in their reenactment for the DREAM protocol with the end goal that a $\mathrm{MH}$ has an arbitrary course at each recreation clock tick, yet a consistent speed amid the whole reproduction time frame. The mobility model in Ko's reenactment for the LAR routing protocol [13] enables MHs to move along a way which is comprised of a few sections. The portion lengths are exponentially conveyed and the 
course of each fragment is arbitrarily picked. Speed is conveyed consistently between . In Das' model [7], a hub picks its speed, course and separation in light of a pre-characterized conveyance, at that point ascertains its next goal and an opportunity to achieve it. At the point when the hub achieves that point, it ascertains another goal and era to achieve it once more.

Johnson's Random Waypoint mobility model [12] is additionally an expansion of arbitrary walk. This model breaks the whole development of a $\mathrm{MH}$ into rehashing respite and movement periods. A versatile host first remains at an area for a specific time then it moves to another arbitrary picked goal at a speed consistently disseminated between [0, MaxSpeed]. Perkins [17] and Broch [5] additionally utilize this model. Chiang's Markovian model [6] is another approach to depict the arbitrary movement. States speak to movement headings. The likelihood of keeping up the present state (or moving to another state) is determined in the transit matrix. Once in movement the $\mathrm{MH}$ will probably continue going at the present course and speed. This model is more reasonable than the irregular model.

Further, Sanchez [20] contemplates the relationship among MHs. This relationship exists while MHs move with a similar reason. As should be obvious in a debacle recuperation, or a military sending, a few versatile hosts in all probability move with a typical target. Two illustrations given by Sanchez are the Pursue model, where MHs endeavor to move towards an objective, and the Column model, which speaks to a looking action.

From the above audit we take note of that most existing cell and specially appointed remote mobility models depict autonomous movement conduct. In the following segment we will build up a gathering mobility model for subordinate conduct, which catches both movement reliance after some time ages and a relationship among MHs.

\section{PROPOSED NEW MOBILITY MODEL}

As we mentioned in previous sections, the collaboration among members of the same team is common in an ad hoc network (e.g., searching for a target). This team relationship makes it possible to partition the network into several groups, each with its own mobility behavior.

One of the primary cases of gathering mobility is the Exponential Correlated Random (ECR) model proposed by BBN [4]. The model imitates every conceivable development, including individual and gathering, by modifying the parameters of a movement work. The new position is an element of the past position, to which an irregular deviationis added. The parameters and vary from gathering to gathering. They drive the gatherings into various moving examples. The ECR mobility model requires a total arrangement of (one for each gathering) to characterize the movement of the whole network. The disadvantage is that it is difficult to constrain a given movement design by choosing the parameters.

The gathering mobility model we proposed here is called Reference Point Group Mobility (OEMM) model. Each gathering has a coherent "focus". The inside's movement de-fines the whole gathering's movement conduct, including area, speed, heading, increasing speed, and so on. Subsequently, the gathering direction is dictated by giving a way to the inside. Generally, hubs are consistently appropriated inside the geographic extent of a gathering. To hub, each is doled out reference point which takes after the gathering development. A hub is arbitrarily set in the area of its reference point at each progression. The reference point plot permits free irregular movement conduct for every hub, notwithstanding the gathering movement.

Figure 1 gives a case of a two gathering model. Each gathering has a gathering movement vector. The figure likewise gives a representation of how a hub moves from time tick to First, the reference purpose of a hub moves from to with the gathering movement vector (Here,). At that point the new hub position is created by adding an arbitrary movement vector to the new reference point. Vector has its length consistently circulated inside a specific sweep focused at the reference point and its bearing consistently appropriated between 0 to 360 degree. This irregular vector is free from the hub's past location. The OEMM model characterizes the movement of gatherings unequivocally by giving a movement way for each gathering. A way which a gathering will take after is given by characterizing an arrangement of check indicates along the way relating given time interims. As time passes by, a gathering moves starting with one check point then onto the next on a proceeding with premise. Each time the gathering focus achieves another check point, it figures the new movement vector from present and next check point areas and from the time interim. 
By legitimate choice of check focuses, one can without much of a stretch model numerous practical circumstances, where a gathering must reach predefined goals inside given time interims to achieve its errand. The check point situation document has the advertisement vantage of decoupling the movement design from the model it-self. Numerous techniques can be utilized to produce a situation record, for example, writing in physically, digitizing a course from a guide, utilizing yields from a program or a profile from genuine. The model has the upsides of giving a general and adaptable system for depicting mobility designs, which are undertaking focused and time limited and simple to actualize and check.

By appropriate determination of check point way and beginning gathering area and parameters in the OEMM model, it is anything but difficult to model different mobility applications. In this segment, we il-lustrate the utilization of OEMM in a couple of agent cases.

The primary model is a land segment model (see Figure 2). The whole region is partitioned into a few neighboring locales, with an alternate gathering in every area. This model can be utilized to model a war zone circumstance, where distinctive brigades are completing same operations (e.g., arrive mine pursuit) in various zones. Each gathering is accountable for one parcel. Another application can be expansive scale fiasco recuperation, where diverse paramedic, police, fire fighters groups work in isolated neighborhoods. We call this model an In-Place Group Model. Figure 2 gives a case of four gatherings working in four adjoining regions, with various movement designs.

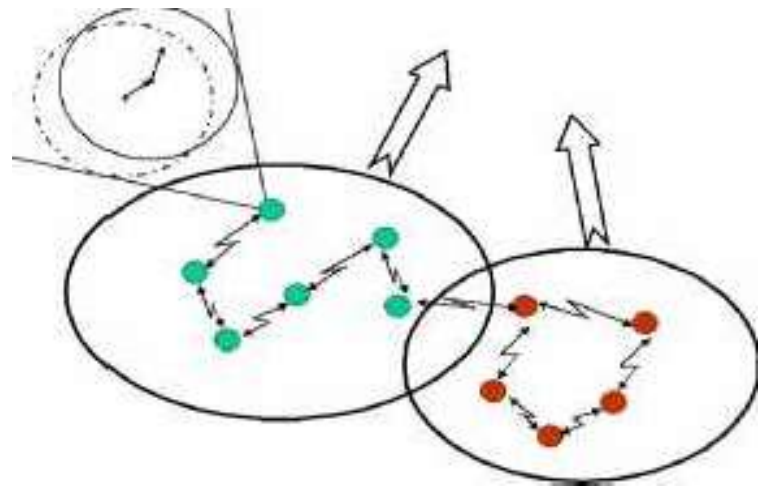

Figure 1: Group Mobility Model

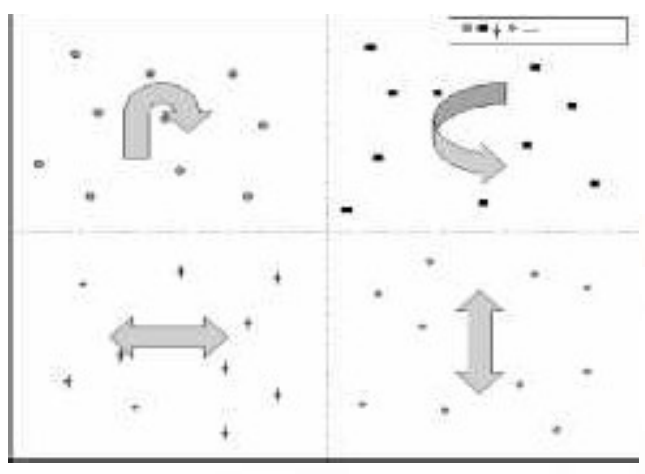

Figure 2: In Place Mobility Model

The second model depicts a covered operation. Diverse gatherings do distinctive errands over a similar region. Nonetheless, the unmistakable necessities of each errand influence their mobility to design very extraordinary. For instance, in a calamity recuperation zone, the safeguard group, the therapeutic right hand group and the analyst group will be haphazardly spread out finished the zone. However, each gathering has a one of a kind movement design, speed, scope and so forth. In Figure 3, there are two gatherings working in a similar zone. We call this model the Overlap Mobility Model.

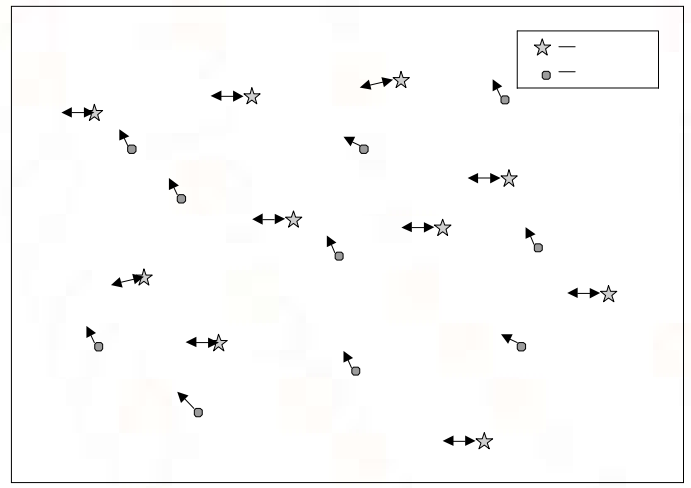

Figure 3: Overlap Mobility Model

The third model is a tradition situation. It models the communication amongst exhibitors and participants. In a tradition, a few gatherings give demos of their examination ventures/items in isolated however associating rooms. A gathering of participants wanders from space to room. They may stop in one space for some time and afterward proceed onward to another room. Or then again, they may go through one room rapidly. Figure 4 demonstrates a gathering of participants wandering around four display rooms. This is known as the Convention Model. Other more perplexing situations which can be modeled with OEMM include: (an) a military move with joint airspecialty, tank and infantry operations. Every benefit 
has an alternate mobility design which can be taken care of with check point way profiles; (b) a two-level mobility model, for instance infantry and helicopters, with moderate and quick hubs, and so on.

A guide can be effortlessly converted into OEMM check point arrange. Subsequently, mobility on interstates can likewise be modeled by OEMM. For instance, the Convention Model could likewise be utilized to mirror the meandering conduct of drivers on a street network.

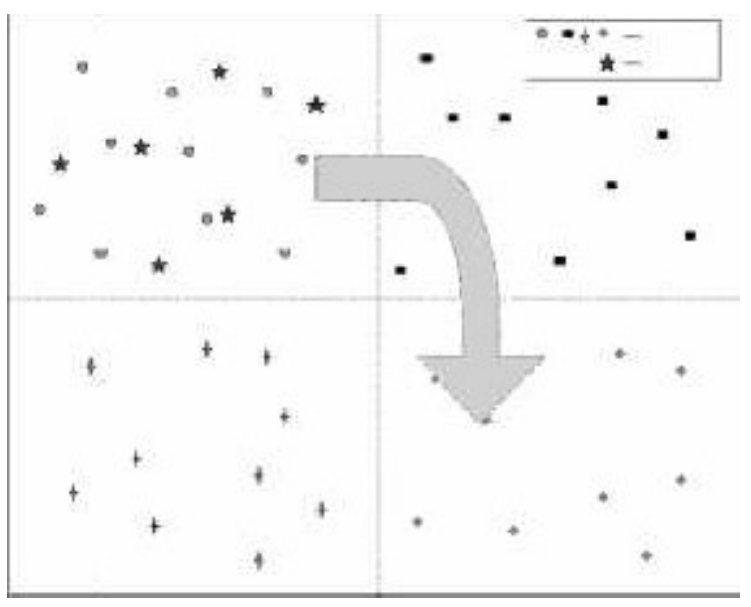

Figure 4: Convention Mobility Model

\section{PERFORMANCE \& PREDICTION OF PROPOSED MOBILITYMODEL}

In a wireless, ad hoc multi-hop network, network, even moderately little developments of the hubs can cause discernible changes in network topology and therefore influence the execution of up-per layer protocols, for example, throughput and deferral. Specially appointed networks are more delicate to mobility than cell wireless networks since in the last the topology changes just when a hub leaves the cell, independent of relative availability with different mobiles. Utilizing the gathering models presented in the past segment, we consider in this segment the effect of mobility on the execution of different structures, protocols and correspondence patterns. Here we characterize a few measurements identified with mobility. We first screen the adjustment in interface status (up, down) caused by the movement of hubs. At the point when two hubs already inside the transmission go (accepting they have same transmission go) move far away, the association is lost. These occasion augments a connection down counter. The other way around, when two hubs move into the transmission go, an association is picked up. This is a connection up case. So we assess how the mobility influences the connection up/down dynamics. Then, we will take a gander at how mobility influences a clustered foundation. As the clusterhead [9] fills in as a local communicate hub crosswise over clusters and as a nearby facilitator of transmissions inside the cluster, we assess the cluster-head change rate. A high clusterhead change rate implies a flimsy network framework for upper layer. Finally, we watch how routing plans will perform under different mobility models. We assess the execution of routing protocols in two ways: (a) conclusion to-end throughput (kbits more than 200-second recreation period) and; (b) control overhead. The control overhead is estimated as megabits every second per cluster in the cluster foundation. With mobility, physically accessible courses may progress toward becoming in-substantial (i.e., may not be found by the routing calculation), making parcels be dropped and prompting throughput debasement and expanding control overhead.

The routing protocols utilized are Destination-Sequence Distance Vector (DSDV) [18], Ad hoc On Demand Distance Vector Routing (AODV) [17], and the Hierarchical State Routing (HSR) [16]. Since just mobility will master duce connect up/down and will influence clustering, the decision of a particular routing protocol has no impact on interface up/down and cluster measurements. Along these lines a basic Bellman Ford routing plan is utilized as a part of the two last trials.

The mobility announced in the execution outlines depends by and large gathering speed and on mean movement dislodging of hubs around their reference focuses.

We utilize a multi-jump, versatile wireless network reproduction model with a clustered framework. The test system is writ-ten in the parallel reenactment dialect Maisie [1]. The net-work comprises of 100 versatile hosts meandering in a 1000x1000 meter square with a reflecting limit. The radio trans-mission run is 120 meters. The information rate is $2 \mathrm{Mb} / \mathrm{s}$. Parcel lengths are $10 \mathrm{kbits}$ for information, $2 \mathrm{kbits}$ for clusterhead neigh-exhausting rundown broadcast, and 500 bits for MAC control bundles. The cradle measure at every hub is 100 bundles. Information parcels are produced following a Poisson procedure with a normal interim of $50 \mathrm{~ms}$. The investigations will transmit a record of 1000 bundles from 10 sources to 10 goals in 200 seconds, and measure the effective throughput with expanding mobility extend. 
We utilize four gatherings in the In-Place Model. The reproduction territory is partitioned into 4 districts as appeared in Figure 2 . Each gathering moves around in one area. We likewise utilize four gatherings in the Overlap Model, however each gathering scrambles over the whole territory. Two of the four gatherings move in a roundabout example various way. One gathering moves straightly, forward and backward. The last gathering is relatively static. In the Convention Model, we have four exhibitor bunches moving gradually in each of the four segments as in the In-Place Model. We additionally have one watcher gathering, which wanders around the whole region. For the investigations with HSR, we pick the settled legitimate subnet estimate, i.e., 25 individuals in each subnet and an aggregate 4 subnets. To make the outcomes com-story, amass setups and ways are indistinguishable for the three routing protocols.

\section{Network Topology}

Figure 5 demonstrates the consequence of the connection up/down examination. At the point when mobility builds, every one of the models demonstrate an expansion in the connection up/down rate. Not surprisingly, the arbitrary mobility model has a higher connection change rate than the gathering mobility models. The Convention Model demonstrates the littlest connection change rate, since the four show bunches move gradually. The In-Place Model and Overlap Model have diverse movement designs, however they have comparative connection change rates.

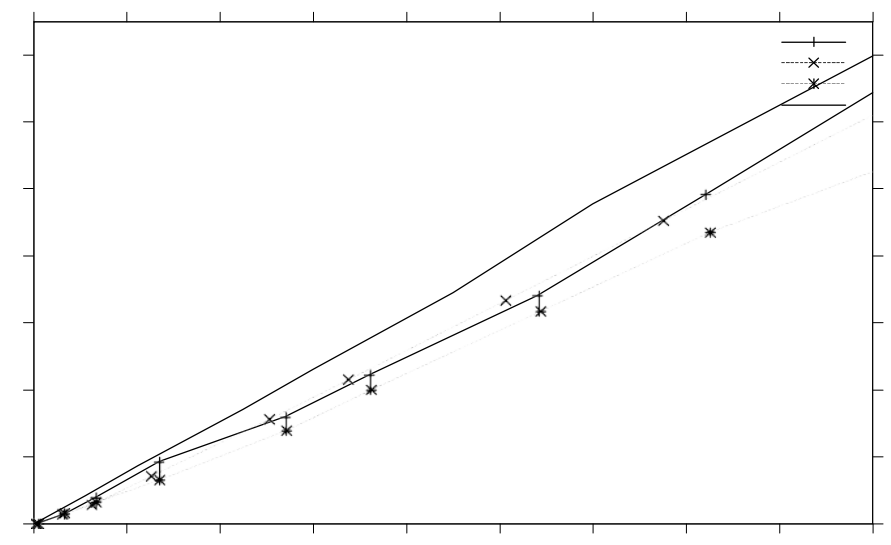

Figure. 5: Link Up/Down Vs. Mobility

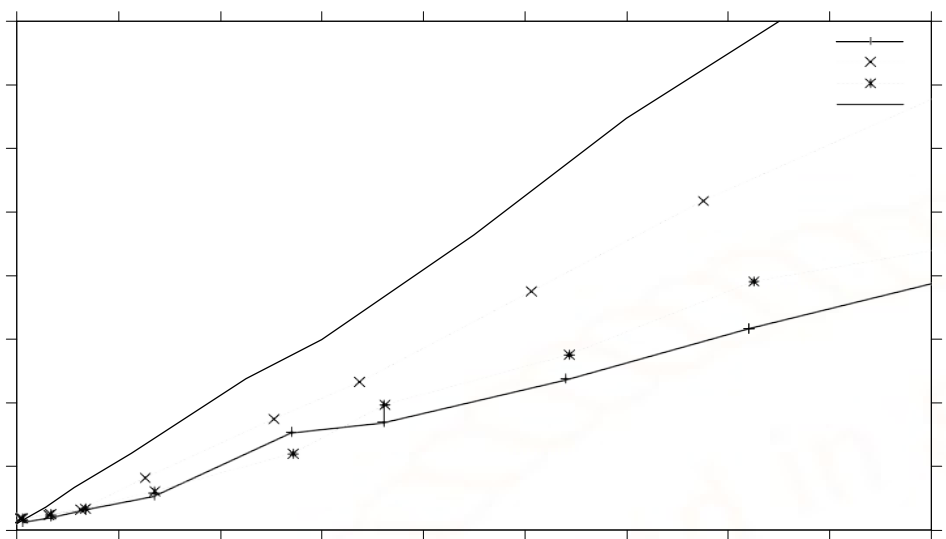

Figure. 6: Cluster Head Changing Vs. Mobility

The after effect of cluster head change rate is appeared in Figure 6. From Figure 6, we can see that the Random mobility model has a higher change rate than the gathering mobility models as already saw in the connection up/down examination. Nonetheless, for this situation distinctive gathering mobility models can effectsly affect the change rate.

In many examinations for execution assessment, we expect that individuals convey arbitrarily crosswise over gathering, with uniform likelihood. In any case, since the very thought of gatherings proposes that the association (i.e., interchanges) is for the most part inside each gathering, it is normal to consider likewise the instance of intra-amass correspondences as it were.

We utilize the Convention Model for this trial, with activity just inside the wandering gathering ("Local Scope Model"). While the wandering gathering interacts with different gatherings or subnets, the individuals from different gatherings will influence its topology and change the interior routing tables. Be that as it may, won't meddle with its activity.

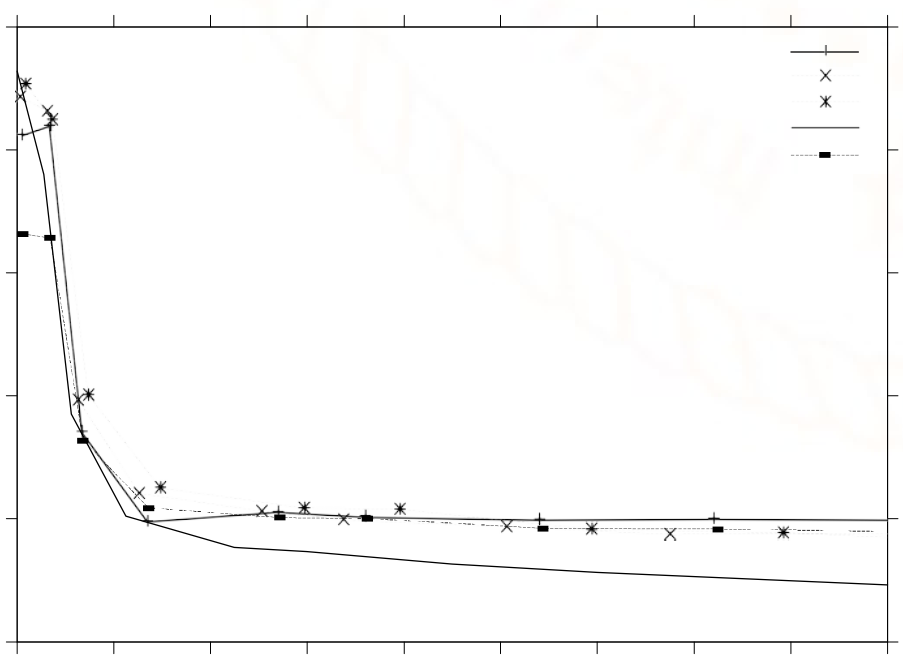

Figure. 7: Throughput of DSDV Vs. Mobility 
In Figure 7, the through put after effects of DSDV are accounted for. DSDV degrades quick when the mobility expands regardless of which mobility model is utilized. The throughput stays at a low level after mobility surpasses $15 \mathrm{~km} / \mathrm{h}$. Of course The Random mobility model is more terrible than the gathering models. DSDV's poor execution is because of overwhelming control message overhead.

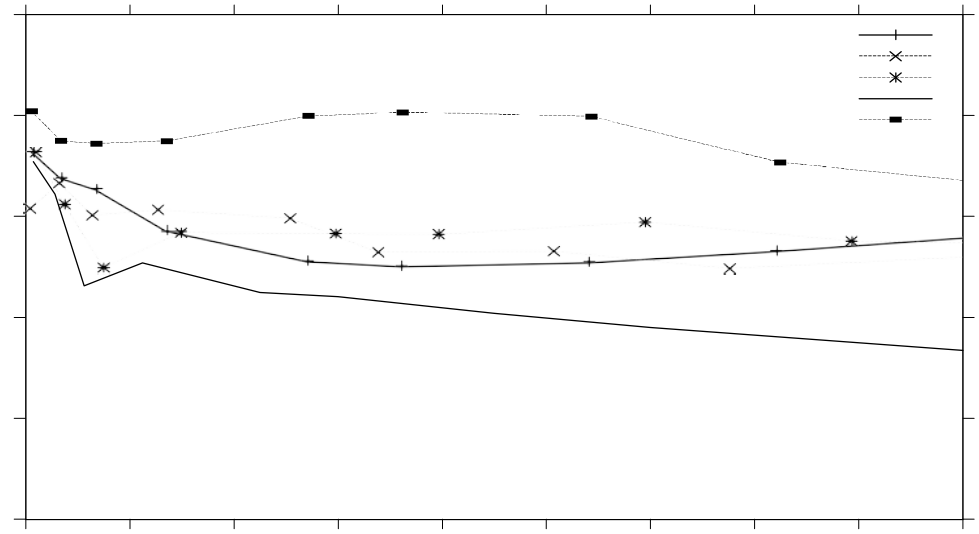

Figure. 8: Throughput of HSR Vs. Mobility

The throughput of HSR is given in Figure 8. The arbitrary mobility model has bring down throughput than the gathering mobility models. This is on account of the subnet various leveled structure of HSR can coordinate well the gathering movement. The throughput of gathering mobility models does not diminish too definitely when the mobility increments, however the clusterhead change rate increments when the mobility increments. This stability comes from the subnet various leveled structure and the home operator offices [16]. At the point when the movement is limited inside a gathering in the Local Scope Model trial, the throughput is the most noteworthy of course.

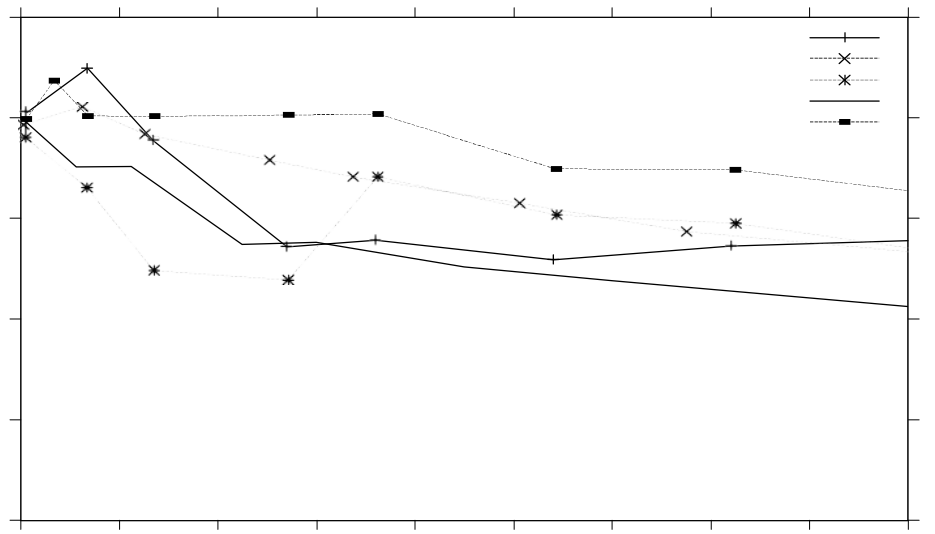

Figure. 9: Throughput of AODV Vs. Mobility
The Above figure 9 repersents about the throughput of the AODV protocols of concern mobility models. When the routing requests and destinations are associated within the roaming group (i.e., Local Scope Model), AODV has very better results. In the remaining models, the sessions are created between arbitrary pairs of nodes across the entire network. Recall that AODV does not maintain background routing tables but it always computes routes for each new request. The performance curves for different mobility patterns are thus more irregular in comparison of those for DSDV and HSR. For the Local Scope Model, performance of AODV is increased by the fact that paths are preserved longer than in the other examples. Thus, routing is more effective and fewer packets are dropped. In general, AODV provides a through- put level comparable to HSR.

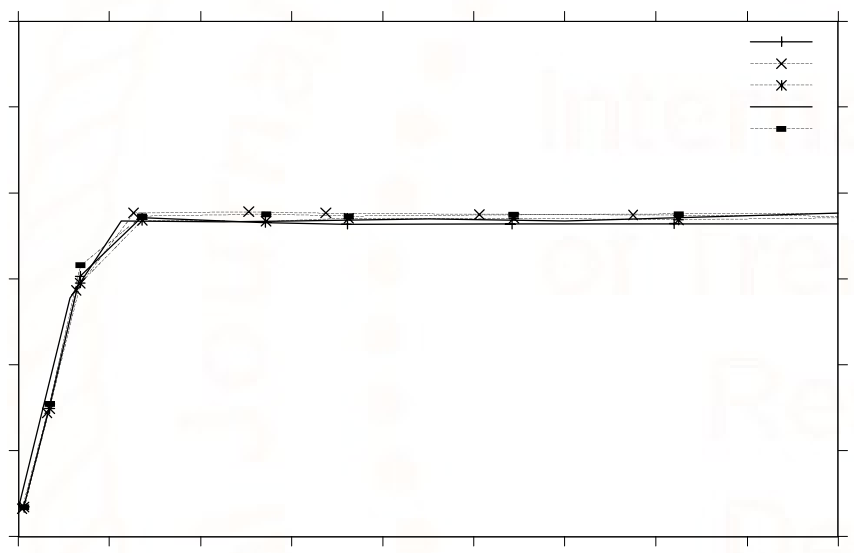

Figure. 10: Control Overhead of DSDV Vs. Mobility

Figure 10 displays the control overhead of DSDV in different mobility models. In Local Scope Model, DSDV has low overhead. It is very sensible as indicated by the situation and is predictable with the high throughput appeared in Figure 9. With the rest of the mobility designs, the over head conduct is reliable with the throughput conduct, i.e., it is more sporadic than those for DSDV and HSR. These examinations demonstrate that the mobility models will influence diverse protocols in various ways. It creates the impression that the Random Model leads to the more regrettable execution much of the time (in any event for the arrangement of routing protocols chose in this examination). Gathering mobility can enhance execution significantly, particularly if the routing protocol can take ad-vantage of a portion of the gathering mobility highlights (similar to the instance of HSR and AODV with Local Scope Model). 
International Journal of Trend in Scientific Research and Development (IJTSRD) ISSN: 2456-6470

\section{CONCLUSION}

In this paper we proposed a group mobility model Reference Point Group Mobility model. The model arranges portable hosts into bunches as indicated by their consistent connection ships. Reproduction comes about demonstrate that the decision of the mobility model has any kind of effect in the physical connection flow and the cluster stability. The Random model creates higher rate of progress in network than amass models. In like manner, Random and Overlap models cause more between blending (than other gathering models) and along these lines more cluster-head changes. Further, extraordinary routing protocols have distinctive responses to the mobility models. In AODV and HSR when interchanges are limited inside the extent of a gathering, the throughput makes strides. DSDV, then again, indicates little affectability to aggregate mobility and to restricted correspondences.

These outcomes demonstrate that, when an ad hoc network is sent in a genuine circumstance, it is not adequate to test it with irregular walk write mobility models since the movement example can interface in a for the most part positive, however in some cases negative path with network protocols. Additionally work is in advance in a few directions. Multicast protocols are being tried, as they remain to get the most effect from bunch mobility. Home Agent plans and Resource Discovery systems are additionally affected by aggregate mobility.

The effect of movement on channel spread must be researched (by utilizing suitable radio proliferation models) keeping in mind the end goal to get a practical match with real proving ground tests. At last, landscape models (slopes, streams, parkways, urban roads, structures, indoor parcel formats, and so forth.) must be represented in that they both con-strain developments and impact the proliferation models.

\section{References:}

[1] R. Bagrodia and W. Liao. Maisie: A language for the design of efficient discrete-event simulations. IEEE Transactions on Software Engineering, 20(4):225-238, 1994.

[2] A. Bar-Noy, I. Kessler, and M. Sidi. Mobile users: to update or not to update? In Proc. of INFOCOM'94 Conference on Computer Communications, pages 570-576, 1994.

[3] S. Basagni, I. Chlamtac, V. R. Syrotiuk, and
B. A. Wood-ward. A distance routing effect algorithm for mobility (DREAM). In ACM/IEEE International Conference on Mobile Computing and Networking (Mobicom98), pages $76-84,1998$.

[4] M. Bergamo et al. System design specification for mobile multimedia wireless network(MMWN) (draft). Technical report, DARPA project DAAB07-95-C-D156, Oct. 1996.

[5] J. Broch, D. A. Maltz, D. B. Johnson, Y.C. $\mathrm{Hu}$, and J. Jetcheva. A performance comparison of multi-hop wire- less ad hoc network routing protocols. In ACM/IEEE International Conference on Mobile Computing and Networking (Mobicom98), pages 85-97, 1998.

[6] C.-C. Chiang. Wireless Network Multicasting. $\mathrm{PhD}$ dissertation, University of California, Los Angeles, Department of Computer Science, May 1998.

[7] S. Das, R. Castaneda, J. Yan, and R. Sengupta. Comparative performance evaluation of routing protocols for mobile, ad hoc networks. In 7th Int. Conf. on Computer Communica- tions and Networks (IC3N), pages 153-161, October 1998.

[8] P. Decker. http://www.comnets.rwthaachen.de/ dpl/thesis/node1.html, 1995.

[9] M. Gerla and J. T.-C. Tsai. Multicluster, mobile, multimedia radio network. ACM/Baltzer Journal of Wireless Networks, 1(3):255-265, 1995.

[10] Z. J. Haas. A new routing protocol for the reconfig- urable wireless networks. In Proc. of IEEE 6th International Conference on Universal Personal Communications (ICUPC'97), pages 562-566, 1997.

[11] Z. J. Haas and B. Liang. Predictive distance based mobility management for PCS networks. In NSF PI's Workshop, Washington, DC, Jan. 1999.

[12] D. B. Johnson and D. A. Maltz. Dynamic Source Routing in ad hoc wireless networks. Kluwer Academic Publishers, 1996.

[13] Y.-B. Ko and N. H. Vaidya. Location-aided routing $(L A R)$ in mobile ad hoc networks.

[14] D. Lam, D. C. Cox, and J. Widom. Teletraffic modeling for personal communications services. 
IEEE Communications Magazine, pages 79-87, February 1997.

[15] K. K. Leung, W. A. Massey, and W. Whitt. Traffic models for wireless communication networks. IEEE Journal on Se-lected Areas in Communications, pages 1353-1364, October 1994.

[16] G. Pei, M. Gerla, X. Hong, and C.-C. Chiang. A wireless hierarchical routing protocol with group mobility. In Pro- ceedings of IEEE WCNC'99, Sep 1999.

[17] C. Perkins and E. Royer. Ad hoc on-demand distance vector routing. In Proceedings of the 2nd IEEE Workshop on Mobile Computing Systems and Applications, pages 90-100, Feb 1999.

[18] C. E. Perkins and P. Bhagwat. Highly dynamic destination- sequenced distance-vector routing(DSDV) for mobile com- puters. In $A C M$ SIGCOMM, pages 234-244, 1994.

[19] I. Rubin and C. W. Choi. Impact of the location area structure on the performance of signaling channels in wireless cellular networks. IEEE Communications Magazine, pages 108-115, February 1997.

[20] M. Sanchez. Mobility models. http://www.disca.upv.es/misan/mobmodel.htm, 1998.

[21] M. M. Zonoozi and P. Dassanayake. User mobility modeling and characterization of mobility patterns. IEEE Journal on Selected Areas in Commonications, 15(7):1239-1252, September 1997. 